\title{
A!
}

This is an electronic reprint of the original article.

This reprint may differ from the original in pagination and typographic detail.

Kaivosoja, Emilia; Sainio, Sami; Lyytinen, Jussi; Palomäki, Tommi; Laurila, Tomi; Kim, Sung I.; Han, Jeon G.; Koskinen, Jari

\section{Carbon thin films as electrode material in neural sensing}

Published in:

Surface and Coatings Technology

DOI:

10.1016/j.surfcoat.2014.07.056

Published: 25/11/2014

Document Version

Peer reviewed version

Please cite the original version:

Kaivosoja, E., Sainio, S., Lyytinen, J., Palomäki, T., Laurila, T., Kim, S. I., Han, J. G., \& Koskinen, J. (2014).

Carbon thin films as electrode material in neural sensing. Surface and Coatings Technology, 259(Part A), 33-38. https://doi.org/10.1016/j.surfcoat.2014.07.056

This material is protected by copyright and other intellectual property rights, and duplication or sale of all or part of any of the repository collections is not permitted, except that material may be duplicated by you for your research use or educational purposes in electronic or print form. You must obtain permission for any other use. Electronic or print copies may not be offered, whether for sale or otherwise to anyone who is not an authorised user. 


\section{Carbon Thin Films as Electrode Material in Neural Sensing}

Kaivosoja Emilia ${ }^{1,2}$, Sainio Sami ${ }^{1}$, Lyytinen Jussi ${ }^{3}$, Palomäki Tommi ${ }^{1}$, Laurila Tomi ${ }^{1}$, Kim Sung I. ${ }^{4}$, Han Jeon G.4 and Koskinen Jari ${ }^{3 *}$

${ }^{1}$ Department of Electrical Engineering and Automation, School of Electrical Engineering, Aalto University, PO BOX 13340, FI-00076 Aalto, Finland

${ }^{2}$ Department of Medicine, Institute of Clinical Medicine, Helsinki University Central Hospital, PO Box 700, 00029 HUS, Finland

${ }^{3}$ Department of Materials Science and Engineering, School of Chemical Technology, Aalto University, PO Box 16200, FI-00076 Aalto, Finland

${ }^{4}$ Institute for Plasma-Nano Materials, Center for Advanced Plasma Surface Technology, Sungkyunkwan University, 300 ChunChun-dong, Jangan-gu, Suwon 440-746, South Korea

For therapeutic purposes, an accurate measurement of dopamine levels in situ would be highly desirable. A novel strategy for the selective determination of dopamine concentrations based on carbon thin film electrodes is presented in this paper. Traditionally, in order to make diamond films conductive, they are doped with different concentration of boron atoms. Here, carbon thin films with varying conductivities were achieved by unbalanced magnetron sputtering. The benefit of the method is that it can be performed at room temperature consequently broadening the selection of suitable substrates. The carbon thin films had a wide potential window, which showed strong dependence on conductivity. The potential window was largest $(4.6 \mathrm{~V})$ with the 
most resistive carbon thin film. On the other hand, the sensitivity of the electrode towards dopamine was not significantly affected by the conductivity. In addition, relatively similar behavior with respect to the dopamine oxidation was observed between various surfaces. The slight differences observed in the electrochemical behavior among the thin films are most likely caused by 1) different conductivities and/or 2) different surface charges and subsequent differences in the chemical properties of the surfaces. In conclusion, it can be stated that a-C thin film is a very potential neural sensing material.

Keywords: Amorphous carbon, potential window, dopamine, electrode *E-mail: jari.koskinen@aalto.fi, tel: +358505956677 


\section{INTRODUCTION}

Neurotransmitters, such as dopamine, are the chemicals that communicate information throughout our brain and body. Abnormal dopamine transmission has been connected with several neurological and psychiatric disorders, e.g. Parkinson's disease, schizophrenia, and Huntington's disease ${ }^{1}$. The accurate measurement of neurotransmitters would provide a better understanding of these diseases and a tool to follow up the output of the treatments. For example, deep brain stimulation (DBS) is increasingly being used as the treatment for Parkinson's disease and feedback control has great potential to improve efficacy, reduce side effects and decrease the cost of the treatment. Most of the attention with this respect has been focused on recording potential biomarkers, such as dopamine, directly from the basal ganglia ${ }^{2}$.

Dopamine can be oxidized by electrochemical methods, and thus they are frequently used for the determination the concentration of dopamine. The primary challenge in dopamine detection is that the concentration of dopamine in the extracellular fluid is low $(0.01-1 \mu \mathrm{M})^{3,4}$, while the amounts of the main detection interferers, which undergo oxidation within the same potential window as dopamine, are several orders of magnitude higher. Secondly, the released dopamine is rapidly cleared from the extracellular space. Accordingly, the sensor must be sensitive, selective and fast. The final challenge is the long term stability, which is confronted by the adsorption of oxidation products leading to the fouling of the electrode.

The rate of the electrochemical reactions is significantly influenced by the nature of the electrode surface. The kinetics of oxygen and hydrogen evolution is significantly slower on

carbon than on most commonly used metal electrodes ${ }^{5}$. The resulting wide potential window is one of the reasons for the widespread use of carbon materials for electrodes. Amorphous 
diamond-like carbon (DLC), is a non-crystalline carbon with high fraction of diamond-like $\left(\mathrm{sp}^{3}\right)$ bonds. Modified DLCs have been applied to various engineering fields, such as magnetic hard disk coatings, wear-protective coatings, razor blades, engine parts, and biomedical coatings ${ }^{6,7}$. DLC coatings are characterized by excellent physical properties (high hardness, high elastic modulus) as well as chemical inertness to any acids, alkaline solutions, and organic solvents ${ }^{8}$. Furthermore, DLC is an attractive electrode material because of its antifouling properties ${ }^{9,10}$. For example, DLC resisted in static conditions adhesion of S. aureus and S. epidermidis compared to titanium, tantalum and chromium ${ }^{11,12,13}$. On the other hand, patterned DLC coatings improved the biocompatibility of silicon surfaces ${ }^{14,15,16}$, making it an attractive material for area-array electrodes.

Traditionally, in order to make diamond films conductive, they are doped with different concentration of boron atoms. Boron doped diamond (BDD) is an excellent electrode material owing to its large potential window in aqueous solution, low background current, fast electron transfer kinetics and stability ${ }^{17}$. These characteristics of diamond electrodes have been employed in a number of applications. Unfortunately, chemical vapor deposition, used for BDD coating, requires high temperatures $\left(>800^{\circ} \mathrm{C}\right)$, which limits the choice of substrate materials.

Recently, $\mathrm{sp}^{2}$ and $\mathrm{sp}^{3}$ hybrid carbon materials, called nanocarbon films, have been developed by employing the electron cyclotron resonance (ECR) sputtering method. This technique provides a nanocrystalline $\mathrm{sp}^{2}$ and $\mathrm{sp}^{3}$ mixed bond structure with an atomically flat surface (surface roughness of $0.05-0.1 \mathrm{~nm}$ ) and high conductivity without doping ${ }^{18}$. Nanocarbon films have the required $\mathrm{sp}^{3}$ bonded carbon structure that exhibits the wide potential window. In addition $\mathrm{sp}^{2}$-carbon is very important for obtaining the electrode activity needed to oxidize the target molecule. These films have been successfully applied for DNA electroanalysis ${ }^{19,}{ }^{20}$. Here 
we present a novel way of making $\mathrm{sp}^{2}$ and $\mathrm{sp}^{3}$ hybrid carbon material with varying conductivity at room temperature and demonstrate its feasibility for electrochemical detection of dopamine.

\section{EXPERIMENT DETAILS}

Four different electrode materials were compared in this study: (i) nanocrystalline conducting carbon (nc-C) and (ii) insulating amorphous carbon films (ta-C, Table 1) on silicon, (iii) silicon itself and (iv) commercial BDD (Windsor Scientific, UK).

\section{1 nc-C coatings}

Conductive carbon films were deposited by a Carbon Film Unbalanced Magnetron (CFUBM) at various target power densities and by adding hydrogen. The substrate materials used was highly doped n++ Si (100). The cylindrical target was Pure graphite (99.99\%) with a diameter of $100 \mathrm{~mm}$. It was discharged with Directly Current power supply. Before deposition, base pressure was reduced $3 \times 10^{-5}$ Torr using diffusion and rotary pump. Prior to deposition the Si wafer was treatment by Ar plasma pre-cleaning with a bias $-700 \mathrm{~V}$ and working pressure 100 mTorr. Working pressure was fixed to 3 mTorr. The carbon film deposition rates are shown in Table I. The deposition rate was controlled by adjusting the target power density in the rage of $10-30$ $\mathrm{W} / \mathrm{cm}^{2}$. The substrate temperature has been measured to be up to $200^{\circ} \mathrm{C}$ depending on the target power and the surface temperature of $270^{\circ} \mathrm{C}$ at the highest was detected.

The coating thickness was kept at $0.5 \mu \mathrm{m}$. A surface profilometer (KLA Tencor Alpha-Step ASIQ) was used to measure the thickness of the carbon films.

\subsection{Control ta-C coatings}


The substrate materials used was highly doped n++ Si (111) wafers (Okmetic). First, the Si wafers were etched in buffered HF solution and dried in flowing nitrogen right before the deposition.

Prior to the coating, the samples were cleaned by argon ion beam sputtering. A $20 \mu \mathrm{m}$ thick layer of titanium was sputtered to enhance the ta-C layer adhesion. ta- $\mathrm{C}$ was deposited using cathodic arc deposition. The capacitor bank of $2.6 \mathrm{mF}$ capacitance was discharged yielding a current pulse with a frequency of $1 \mathrm{~Hz}$, a maximum current of about $3 \mathrm{kA}$ and a half width of $150 \mu \mathrm{s}$. An accumulation of about $1.4 \times 10^{15}$ atoms $\mathrm{cm}^{-2}$ during each pulse was obtained. The sample holder was rotated with the rotation axis perpendicular to the direction of the plasma plume.

\subsection{AFM}

AFM Measurements were done using PSIA XE-100 Advanced Scanning Probe Microscope with MicroMasch type B contact silicon cantilever (nominal radius of tip curvature $<35 \mathrm{~nm}$, tip height $15-20 \mu \mathrm{m}$, tip cone angle $<20^{\circ}$, resonant frequency $105 \mathrm{kHz}$, force constant $2.0 \mathrm{~N} / \mathrm{m}$ ) using tapping mode. AFM scan rate was $1 \mathrm{~Hz}$ and the scanned areas were between $1-100 \mu \mathrm{m}$ in height and width.

\subsection{Resistivity of nanocrystalline coatings}

The electrical resistivity of the carbon film was measured by 4-point probe (CMT-SR-1000N). The probe current was in the range from $10 \mathrm{nA}$ to $100 \mathrm{~mA}$.

\subsection{Cyclic voltammetry}


Cyclic voltammetry measurements were made using a potentiostat (QuadStat, eDAQ Pty Ltd, Denistone East, Australia) attached to a recording unit (e-corder 821, eDAQ) and computer. The Echem software (ADInstruments Pty ltd, Castle Hill, Australia) was used to enter the parameters of the experiment and control the potentiostat. A titanium rod with $2.5 \mu \mathrm{m}$ thick coating of platinum (ET078, eDAQ) served as the counter electrode and a commercial $\mathrm{Ag} / \mathrm{AgCl}$ electrode (Sarissa Biomedical Ltd., Coventry, UK) was used as the reference. All measurements were made in a Faraday cage (VistaShield, Gamry Instruments, Warminster, USA) to avoid electrical interference.

The potential window was determined in nitrogen purged 0.15 M H2SO4 (Merck KGaA, Darmstadt, Germany) with $\mathrm{pH} 0.55$ at a scan rate of $400 \mathrm{mV} / \mathrm{s}$. Then the potential was cycled approximately 20 to 25 times until a steady-state $\mathrm{CV}$ was obtained. The capacitive currents were measured at $-0.15 \mathrm{~V}$ vs $\mathrm{Ag} / \mathrm{AgCl}$ in $\mathrm{H} 2 \mathrm{SO} 4$ from steady state $\mathrm{CVs}$.

The ability to detect dopamine was assessed with cyclic voltammetry in nitrogen purged phosphate buffered saline (PBS) with $5 \mathrm{nM}-100 \mu \mathrm{M}$ dopamine (Sigma-Aldrich, St. Louis, USA) at a scan rate of $400 \mathrm{mV} / \mathrm{s}$. The initial potential was chosen as $0 \mathrm{~V}$, since no redox reactions occurred at that potential. Three cycles were scanned at each dopamine concentration.

\section{RESULTS AND DISCUSSION}

\subsection{Surface characteristics}

High concentration of $\mathrm{sp}^{2}$ in the nc-C and high concentration of $\mathrm{sp}^{3}(80-85 \%)$ in ta-C films was

detected by applying XPS, Raman and TEM as reported earlier ${ }^{21,22}$. The nc-C films presented a 
smooth surface, with an average surface roughness of 1-3 nm (Table Figure 1). The roughness depends on the deposition parameters described in more detail elsewhere ${ }^{23}$. Table 1 shows the measured resistivity of the samples. The most conductive surface has a resistivity of $0.03 \Omega \mathrm{cm}$ whereas the hydrogenated carbon and control ta-C are practically insulators.

\subsection{Electrochemical performance}

Water window is the potential range between hydrogen evolution, where proton reduction occurs, and oxygen evolution, where water oxidation occurs. A wide water window is desirable because it enables the greatest degree of analyte characterization in water based solutions.

The electrochemical performance of the carbon thin film electrodes was excellent (Figure 1). The potential window was very wide for all of the materials and the capacitive background current low (Table 2). The width of the water window was strongly dependent on the conductivity, the widest window being on the most resistive surface and the narrowest window on the least resistive surface. This is somewhat expected as the $\mathrm{sp}^{3}$ to $\mathrm{sp}^{2}$ ratio changes along with the conductivity. This is in agreement with previous observations that diamond electrodes $\left(\right.$ high $\left.\mathrm{sp}^{3}\right)$ have electrochemical windows significantly wider than most graphitic $\left(\mathrm{sp}^{2}\right)$ materials ${ }^{24}$. Double layer capacitance was determined from the $\mathrm{CV}$-curves in $\mathrm{H}_{2} \mathrm{SO}_{4}$ for each of the electrode material. Geometric area of the electrode was used in the calculations and the difference between the anodic and cathodic current densities was used $(\Delta i=2 \times C \times v)$. Cycling speed was 400 $\mathrm{mV} / \mathrm{s}$. The results obtained are presented in Table 2 . The capacitance values show a decreasing trend as a function of resistivity starting from sample nc-C2. Thus, as the resistivity increases the capacitance value decreases. Electrode nc-C1 is an exception from this trend and shows lower than expected value. The reason for this behavior is at the moment not unambiguously known. 
The capacitance values are relatively large when compared to typical values obtained in the literature for different carbon materials. Lowest reported values for DLC are $3.45 \mu \mathrm{F} / \mathrm{cm}^{2}$ for nitrogen doped DLC fabricated by sputtering and $1.02 \mu \mathrm{F} / \mathrm{cm}^{2}$ for DLC fabricated by Filtered Cathod Vacuum Arc (FCVA) method ${ }^{25},{ }^{26}$. Typical values reported for boron doped diamond (BDD) electrodes vary between 3.7 and $7.1 \mu \mathrm{F} / \mathrm{cm}^{2}{ }^{27}$ and that for glassy carbon is generally given to be about $30 \mu \mathrm{F} / \mathrm{cm}^{2}{ }^{28}$. Thus, especially the value for ta-C $\left(105 \mu \mathrm{F} / \mathrm{cm}^{2}\right)$ is surprisingly large. This may be related to the fact that in our case there are no really clear flat double layer regions (Fig. 1), as there are small but continuous increase in current as a function of potential throughout the "linear" region practically with all the electrode materials. This may indicate that there are oxygen containing groups present at the electrode surfaces or that some surface reactions and/or IR drop contributes to the measured "double layer" capacitance.As a reference to the above electrochemical properties, the water window of silicon was extremely wide $(5 \mathrm{~V})$, whereas that of the BDD was $2.8 \mathrm{~V}$. The capacitance value of the BDD as determined here was a bit higher than expected.

\subsection{Detection of dopamine}

Carbon thin films improved the sensitivity of the electrode towards dopamine as silicon itself could not detect dopamine at all. Despite the large difference in potential window, there was practically no difference in the sensitivity towards dopamine between the carbon electrodes. All of the carbon surfaces are capable of detecting $100 \mu \mathrm{M}$ dopamine, except the hydrogenated nc-C, which only detected $1 \mathrm{mM}$ dopamine (Figure 2). Notably, the background current decreased with increasing resistivity. This is consistent with the results from water window determination. It is to be noted that, owing to the smaller water window of the nc-C1 as shown in Fig. 1 narrower potential range for DA detection was used in these measurements. Thus, the fact that only one 
reduction and oxidation peak is visible in Fig. 2 for this material stems from this and does not indicate that DA would behave differently on top of this material. The main differences that can be observed between the carbon electrodes are (i) the decrease in the dopamine oxidation current (and current density as all the electrodes (except BDD) had the same area) and (ii) shift in the oxidation potential to more anodic values, both as a function of increased resistivity. We will consider next the behavior of dopamine on top of these carbon based electrodes in a bit more detail. Several electrochemical pathways have been proposed for dopamine including ECE, ECC and even ECECEE mechanisms (E stands for an electrochemical reaction and $\mathrm{C}$ for a chemical reaction $)^{29}$. In the voltammograms of Figure 2 the first electrochemical step between dopamine and its oxidation product dopaminequinone is always observed. This step corresponds to the second oxidation peak at around $1 \mathrm{~V}$ and the first reduction peak at around $-0.1 \mathrm{~V}$. Dopaminequinone can undergo a chemical reaction by intramolecular cyclization at physiological $\mathrm{pH}$ leading to leucodopaminechrome. Leucodopaminechrome can further oxidize to dopaminechrome, which takes place immediately as the overpotential at this part of the anodic scan is very high. This second electrochemical step causes another oxidation and reduction peak to appear in the case of nc-C2, nc-C3, nc-C4, nc-C5, ta-C and BDD. Again it is to be noted that the narrower potential range used for nc-C1 prevents us from observing these peaks.

Dopamine has been shown to adsorb on the carbon electrode surface ${ }^{30}$. The interactions between the carbon surface and the adsorbate that govern adsorption include dipole-dipole interactions, induced dipoles, hydrophobic and electrostatic effects and covalent bonding. These interactions will be affected by the carbon allotrope used, its preparation, the exposure of basal and edge planes, and surface oxides ${ }^{5}$. Previously it has been speculated that dopamine oxidation is significantly slower on diamond than on glassy carbon because of the weak adsorption ${ }^{24,31}$. 
This speculation was derived from the observation that the rate of electron transfer at $\mathrm{sp}^{2}$ carbon electrodes is mediated by surface carbonyl functionalities; however, this inner-sphere, catalytic pathway is absent on diamond ${ }^{31}$.

The adsorption of DA may increase the oxidation current significantly. As this was indeed observed to vary between the samples, question remains, whether this is mainly due to the effect of electrical properties, surface topography or surface chemistry. According to AFM results, the surfaces of all the carbon thin film samples were rather similar. Moreover, BDD consists of crystals and facets whereas the surface of carbon thin films is very smooth. This would indicate that the electrical or chemical properties, rather than topography, cause the small differences in electrochemical behavior. The thickness of the coating did not have a marked role in the electrochemical performance of the electrodes. nc-C3 and nc-C4 show similar reaction kinetics to ta-C despite the large difference in the thickness (500 $\mathrm{nm}$ and $30 \mathrm{~nm}$, respectively). This implies that the "bulk" conductivity of the thin film is not the key element in this respect. In addition to resistivity, surface charge may play a relevant role on dopamine adsorption. It has been observed that the extent of adsorption of ionic analytes can vary strongly with surface charge $^{32}$. One explanation for this is that the surface charge may influence in which orientation dopamine arrives on the surface, at least at the open circuit potential. Thus, the electron emission properties and density of states of the thin carbon film surfaces may be in the central role here. Different diamond films have been shown to carry different surface charges. For example, the zeta-potential for laser-ablated DLC was higher $(-54.5 \pm 0.6 \mathrm{mV})^{13}$ than for DLC film fabricated using filtered pulsed arc discharge $(-70.2 \pm 1.1 \mathrm{mV} \text {, both measured in } 1 \mathrm{mM} \mathrm{KCl}, \mathrm{pH} 7)^{15}$. This will most likely be reflected also as differences in the surface chemistries between the two samples, which could then translate into different electrochemical behavior during cyclic 
voltammetry. Moreover, it has been suggested that the lack of hydrogen bonding sites on a given carbon based electrode surface may hinder the adsorption of dopamine and slow down the oxidation reaction considerably ${ }^{31}$. It is likely that the diamond film surfaces exhibit differences in the carbon - oxygen bonding components, e.g. carboxyl and hydroxyl structures. To find out if there is some difference in the hydrogen bonding capability among the surfaces, detailed surface analyses must be carried out. Before these have been carried out, the question about the role of chemistry of the surface must remain open.

The different adsorption behavior is highly relevant in two aspects: 1) stronger adsorption correlates with-may lead to increased sensitivity but 2) simultaneously the adsorption kinetics may limit the electrode's response to rapid concentration changes ${ }^{30}$.

\subsection{The effect of scan rate}

Finally, the effect of different scan rate was investigated. For reversible electron transfer, peak separation value $(\Delta \mathrm{Ep})$ is independent of the scan rate. However, Figure 3 shows that $\Delta \mathrm{Ep}$ changes as the scan rate is altered, which implies that the heterogeneous electron transfer kinetics are slow at the carbon thin film electrode surfaces. Moreover, repetitive cycling resulted in decreased peak currents (Figure 3). This indicates that some changes (passivation) occur on the coating surface. It has been previously suggested ${ }^{31}$ that with scanning rates higher than about 50 $\mathrm{mV} / \mathrm{s}$ the chemical reaction that the dopamine-quinone can experience has no time to occur. When one looks at the voltammograms in Figure 3 two trends are more or less clear: (i) the fact that the peak potentials do not change as a function of number of cycles indicates that the electrochemical reaction constant does not change and (ii) the decreasing peak current on the other hand indicates that the active surface area of the electrode is continuously reduced. Thus it 
seems that with the high cycling speeds used here a passivating surface film completely covering the electrode is not formed, but rather only part of the surface is passivated. Previously, it has been shown that for example on gold electrodes part of the dopaminechrome formed is adsorbed on the surface of the electrode and undergoes further chemical reaction, which leads to the

formation of a passivating surface polymer film, contributing to the decrease in peak height ${ }^{29}$. A detailed study on the dopamine behavior on single crystal Pt electrodes with different orientations has also pointed out formation of polymer films on these materials ${ }^{33}$. In our case it may be that the polymer layer does not have time to form owing to the high cycling speed and only part of the surface is covered with adsorbed monomers or oligomers.

The wide water window indicates high prospect of the selective detection of dopamine. However, the sensitivity of the carbon thin film electrodes needs to be further improved. This could be achieved by implementing functional carbon nanostructures that provide chemically active spots that improve the sensitivity of the sensor. We have previously shown this with DLCplatinum composite electrodes ${ }^{22}$.

\section{CONCLUSION}

A novel strategy for the selective detection of dopamine based on the carbon thin film electrode has been presented in this paper. It was observed that the carbon thin films are very potential neural sensing materials. These materials have a large water window and a low background signal (capacitive current). The water window was strongly depended on the conductivity, whereas sensitivity towards dopamine did not depend on conductivity. The detection limit of dopamine was $100 \mu \mathrm{M}$ for all of the tested materials, except for the hydrogenated nc-C, which only detected $1 \mathrm{mM}$ of dopamine. The sensitivity towards dopamine was not influenced by the 
conductivity of the material significantly. In addition, all the materials showed relatively similar behavior with respect to dopamine. The apparent difference between nc-C1 and the other electrode materials was caused most likely by the narrower potential window used for that specific material. The observed change in the dopamine oxidation current between the samples was most likely caused by differences in surface charge and chemistry.

The carbon thin films showed similar reaction kinetics to BDD electrode. However, the applicability of the BDD is limited because of the high-temperature requiring fabrication process. This novel strategy for the selective detection of dopamine based on the carbon thin film electrode has certain advantages compared to BDD electrodes although the sensitivity needs to be further improved.

Acknowledgments: The research was funded by Aalto University strategic funding, Academy of Finland, the Finnish Funding Agency for Technology and Innovation (TEKES) project Hybrid Carbon Nanomaterial Interface Between Living and Electronic Worlds (HILE) and Korean National Science Foundation. 


\section{References}

1. J.M. Beaulieu, and R.R. Gainetdinov, Pharmacol. Rev. 63, 182 (2011).

2. S. Little, and P. Brown, Ann. N. Y. Aca. Sci. 1265, 9 (2012).

3. J.M. Zen, and P.J. Chen, Anal. Chem. 69, 5087 (1997).

4. R.M. Wightman, L.J. May, and A.C. Michael, Anal. Chem. 60, 769A (1988).

5. R.L. McCreery, Chem. Rev. 108, 2646 (2008).

6. C. Casiraghi, J. Robertson, and A.C. Ferrari, Materials Today 10, 44 (2007).

7. R. Hauert, Diam. Relat. Mater. 12, 583 (2003).

8. V. Rehacek, I. Hotovy, and M. Vojs, Sens. Act. B 127, 193 (2007).

9.T. Goto, T. Yasukawa, K. Kanda, S. Matsui, and F. Mizutani, Anal. Sci. 27, 91 (2011).

10. K. Page, M. Wilson, and I.P. Parkin, J Mater. Chem. 19, 3819 (2009).

11. J. Levon, K. Myllymaa, V.P. Kouri, R. Rautemaa, T. Kinnari, S. Myllymaa, Y.T. Konttinen, R. Lappalainen, J. Biomed. Mater. Res. A. 92, 1606 (2010). doi: 10.1002/jbm.a.32486.

12. K. Myllymaa, J. Levon, V.M. Tiainen, S. Myllymaa, A. Soininen, H. Korhonen, E. Kaivosoja, R. Lappalainen, and Y.T. Konttinen, Coll. Surf. B. 101, 290 (2013).

13. E. Kaivosoja, S. Virtanen, R. Rautemaa, R. Lappalainen, and Y.T. Konttinen, Eur. Cell. Mater. 24, 60 (2012).

14. E. Kaivosoja, S. Myllymaa, V Kouri, K. Myllymaa, R. Lappalainen, and Y.T. Konttinen, Eur. Cell. Mater. 19, 147 (2010).

15. E. Kaivosoja, S. Myllymaa, Y. Takakubo, H. Korhonen, K. Myllymaa, Y.T. Konttinen, R. Lappalainen, and M. Takagi, J. Biomater. Appl. 27, 862 (2013).

16. S. Myllymaa, E. Kaivosoja, K. Myllymaa, T. Sillat, H. Korhonen, R. Lappalainen, and Y.T. Konttinen, J. Mater. Sci. Mater. Med. 21, 329 (2010). 
17. Yu.V. Pelskov, A.Ya. Sakharova, M.D. Krotova, L.L. Bouilov and B.V. Spitsyn, J. Electroanal. Chem. 228, 19 (1987).

18. O. Niwa, J. Jia, Y. Sato, D. Kato, R. Kurita, K. Maruyama, K. Suzuki, and S. Hirono, J. Am. Chem. Soc. 128, 7144 (2006).

19. K. Goto, D. Kato, N. Sekioka, A. Ueda, S. Hirono, and O. Niwa, Anal. Biochem. 405, 59 (2010).

20. D. Kato, N. Sekioka, A. Ueda, R. Kurita, S. Hirono, K. Suzuki, and O. Niwa, J. Am. Chem. Soc. 130, 3716 (2008).

21. H.S. Myung, Y.S. Park, B. Honga, J.G. Han, Y.H. Kim, J.Y. Leeb, and L.R. Shaginyan. Thin Solid Films 494, 123 (2006).

22. T. Laurila, A. Rautiainen, S. Sintonen, H. Jiang, E. Kaivosoja, J. Koskinen, and M. PaulastoKröckel, Mater. Sci. Eng. C. 34, 446 (2014). doi: 10.1016/j.msec.2013.09.035

23. H.S. Myung, Y.S. Park, M.J. Jung, B. Hong, and J.G. Han. Materials Letters 58,1513 (2004).

24. R.L. McCreery, Chem. Rev. 108, 2646 (2008).

25. M.A. Méndez, B. Su, and H.H. Girault, J. Electroan. Chem 634, 82 (2009).

25. A. Zeng, E. Liu, S.N. Tan, S. Zhang, and J. Gaoa. Electroanalysis 14, 1110, (2002).

26. E. Liu, and H.W. Kwek. Thin Solid Films 516, 5201 (2008).

27. G.M. Swain, and A. Ramesham A. Anal. Chem. 65, 345 (1993).

28. M.T. McDermott, C.A. McDermott, and R.L. McCreery. Anal. Chem. 65, 937 (1993).

29. Y. Li, M. Liu, C. Xiang, Q. Xie, and S. Yao. Thin Solid Films 497, 270 (2006).

30. B.D. Bath, D.J. Michael, B.J. Trafton, J.D. Joseph, P.L. Runnels, and R.M. Wightman, Anal. Chem. 72, 5994 (2000).

31. M.C. Granger, M. Witek, J. Xu, J. Wang, M. Hupert, A. Hanks, M.D. Koppang, J. E. Butler, 
G. Lucazeau, M. Mermoux, J.W. Strojek, and G.M. Swain, Anal. Chem. 72, 3793 (2000).

32. C.D. Allred, and R.L. McCreery, Anal. Chem. 64, 444 (1992).

33. S. Chumillas, M. Figueireido, V. Climent, and J. Feliu. Electrochemica Acta 109, 577 (2013). 
Table I Coating conditions, resistivity and surface roughness of the samples. For comparison, the surface roughness of the commercial BDD electrode is given.

\begin{tabular}{|c|c|c|c|c|c|}
\hline Sample & $\begin{array}{c}\text { Coating } \\
\text { thickness }(\mathbf{n m})\end{array}$ & $\begin{array}{c}\text { Working pressure } \\
\text { (mTorr) }\end{array}$ & $\begin{array}{c}\text { Deposition rate } \\
(\mathrm{nm} / \mathrm{min})\end{array}$ & Resistivity & $\begin{array}{c}\text { Surface } \\
\text { roughness }(\mathbf{n m})\end{array}$ \\
\hline nc-C1 & 500 & 3 & 120 & $0.03 \Omega \mathrm{cm}$ & 2.5 \\
\hline $\mathrm{nc}-\mathrm{C} 2$ & 500 & 3 & 36 & $3.60 \Omega \mathrm{cm}$ & 1.2 \\
\hline nc-C3 & 500 & 3 & 18 & $60 \Omega \mathrm{cm}$ & 1.1 \\
\hline $\mathrm{nc}-\mathrm{C} 4$ & 500 & 3 & 3 & $200 \Omega \mathrm{cm}$ & 2.8 \\
\hline nc-C5 & 500 & $3\left(\mathrm{H}_{2} 7 \%\right.$ mixing $)$ & 105 & $5 \times 10^{6} \Omega$ & 1.6 \\
\hline ta-C & 30 & 0.01 & 6 & $\gg 10^{6} \mathbf{\Omega c m}$ & 0.33 \\
\hline BDD & & & & & 1.0 \\
\hline
\end{tabular}


Table II Water window and the double layer capacitances of the samples.

\begin{tabular}{cccc}
\hline Sample & $\begin{array}{c}\text { Potential range }(\mathbf{m V})\left(\mathbf{H}_{2} \mathbf{S O}_{4}\right) \\
\text { Lower }\end{array}$ & Capacitance $\left(\boldsymbol{\mu F} / \mathbf{c m}^{2}\right)$ \\
\hline nc-C1 & -250 & 1500 & $46 \pm 1$ \\
\hline nc-C2 & -1300 & 1700 & $83 \pm 6$ \\
\hline nc-C3 & -1800 & 2000 & $60 \pm 7$ \\
\hline nc-C4 & -1600 & 1600 & $52 \pm 7$ \\
\hline nc-C5 & -2200 & 2400 & $37 \pm 9$ \\
\hline Si & -2500 & 2500 & $0.5 \pm 0.3$ \\
\hline ta-C & -1700 & 2000 & $105 \pm 7$ \\
\hline BDD & -1100 & 1800 & $33 \pm 6$ \\
\hline
\end{tabular}




\section{Figures}


Potential (V vs $\mathrm{Ag} / \mathrm{AgCl}) \quad$ Potential ( $\mathrm{V}$ vs $\mathrm{Ag} / \mathrm{AgCl}) \quad$ Potential (V vs $\mathrm{Ag} / \mathrm{AgCl}) \quad$ Potential (V vs $\mathrm{Ag} / \mathrm{AgCl})$

Figure 1 Water windows of the samples. The current range of BDD is different to others because of the size difference of the electrodes. 

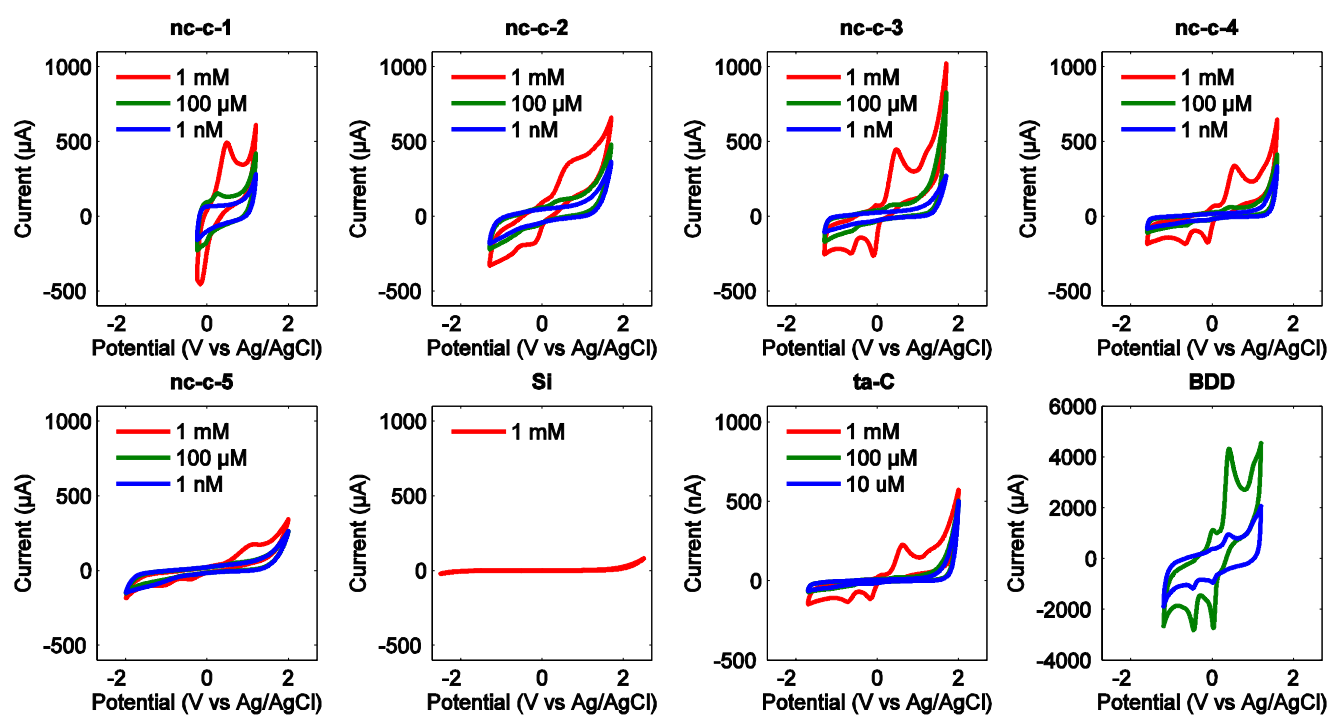

Figure 2 Dopamine detection. The current range of BDD is different to others because of the size difference of the electrodes. 

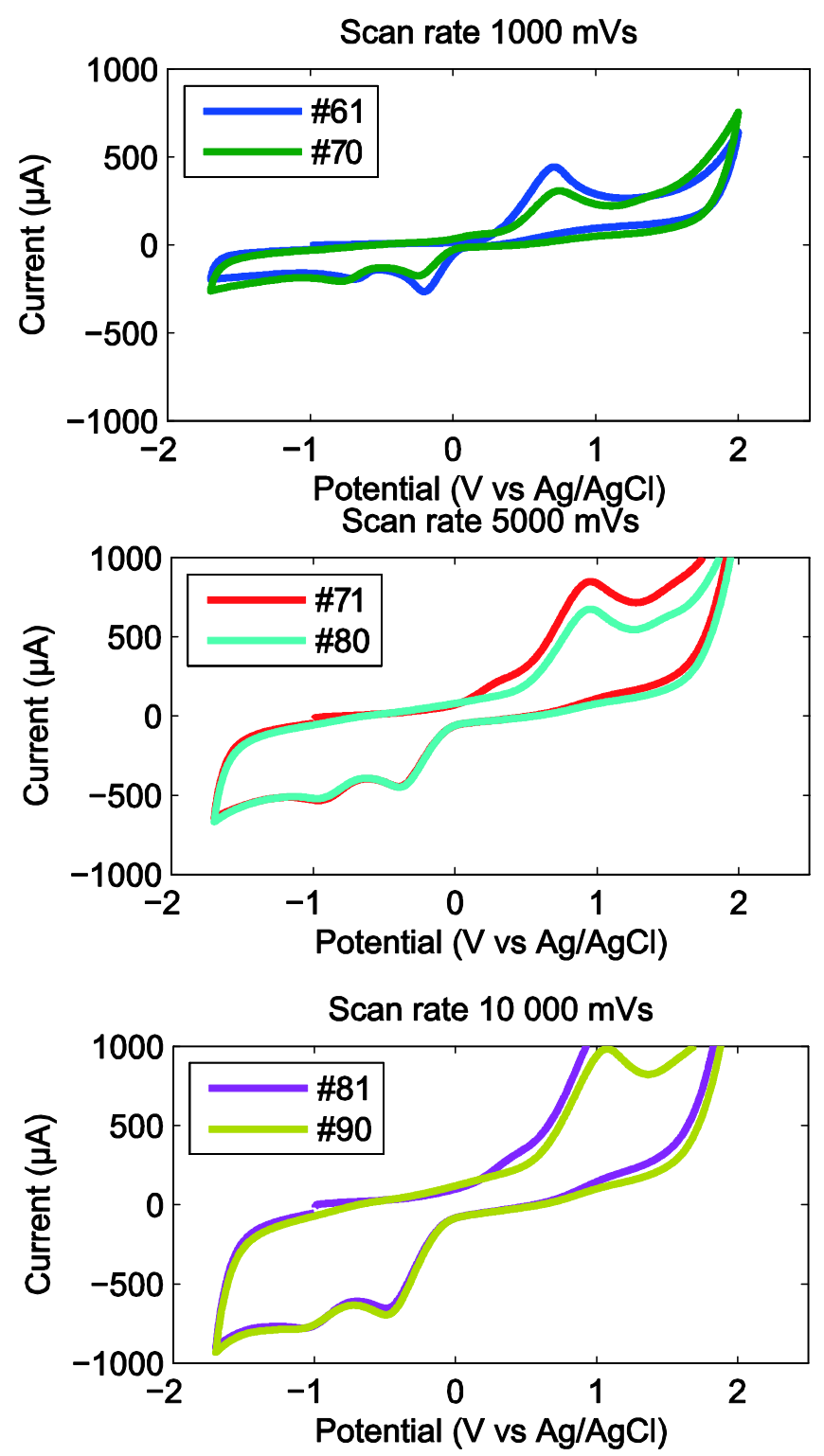

Figure 3 The effect of scan rate. $1 \mathrm{mM}$ dopamine concentration. The number of scanning cycles has been indicated in the legend. 
Table 1. Coating conditions, resistivity and surface roughness of the samples. For comparison, the surface roughness of the commercial BDD electrode is given.

\begin{tabular}{|c|c|c|c|c|c|}
\hline Sample & $\begin{array}{c}\text { Coating } \\
\text { thickness } \\
(\mathrm{nm})\end{array}$ & $\begin{array}{l}\text { Working } \\
\text { pressure } \\
\text { (mTorr) }\end{array}$ & $\begin{array}{c}\text { Deposition rate } \\
\text { (nm/min) }\end{array}$ & Resistivity & $\begin{array}{c}\text { Surface } \\
\text { roughness } \\
(\mathrm{nm})\end{array}$ \\
\hline nc-C1 & 500 & 3 & 120 & $0.03 \Omega \mathrm{cm}$ & 2.5 \\
\hline nc-C2 & 500 & 3 & 36 & $3.60 \Omega \mathrm{cm}$ & 1.2 \\
\hline nc-C3 & 500 & 3 & 18 & $60 \Omega \mathrm{cm}$ & 1.1 \\
\hline nc-C4 & 500 & 3 & 3 & $200 \Omega \mathrm{cm}$ & 2.8 \\
\hline nc-C5 & 500 & $\begin{array}{l}3\left(\mathrm{H}_{2} 7 \%\right. \\
\text { mixing })\end{array}$ & 105 & $5 \times 10^{6} \Omega$ & 1.6 \\
\hline ta-C & 30 & 0.01 & 6 & $>>10^{6} \Omega \mathrm{cm}$ & 0.33 \\
\hline BDD & & & & & \\
\hline
\end{tabular}

Table 2. Water window and the double layer capacitances of the samples.

\begin{tabular}{|l|l|l|l||}
\hline \multicolumn{1}{||l|}{ Sample } & \multicolumn{1}{|c||}{ Potential range $(\mathbf{m V})\left(\mathrm{H}_{2} \mathrm{SO}_{4}\right)$} & Capacitance $\left(\boldsymbol{\mu F} / \mathbf{c m}^{2}\right)$ \\
\hline \hline & Lower & Upper & \\
\hline \hline nc-C1 & -250 & 1500 & $46 \pm 1$ \\
\hline \hline nc-C2 & -1300 & 1700 & $83 \pm 6$ \\
\hline \hline nc-C3 & -1800 & 2000 & $60 \pm 7$ \\
\hline nc-C4 & -1600 & 1600 & $52 \pm 7$ \\
\hline \hline nc-C5 & -2200 & 2400 & $37 \pm 9$ \\
\hline Si & -2500 & 2500 & $0.5 \pm 0.3$ \\
\hline ta-C & -1700 & 2000 & $105 \pm 7$ \\
\hline \hline BDD & -1100 & 1800 & $33 \pm 6$ \\
\hline
\end{tabular}

